\title{
Which way to the next frontier? Finding the most powerful cluster lenses
}

\author{
Harald Ebeling ${ }^{1}$ and the eMACS team \\ ${ }^{1}$ Institute for Astronomy, University of Hawaii, Honolulu, HI 96822 \\ email: ebeling@ifa.hawaii.edu
}

\begin{abstract}
While the identification of a worthy topic or challenge is fundamental to the goal of conducting cutting-edge research, another ingredient is indispensable in the field of observational astronomy: the best possible target. Indeed, the ability to choose from a significant number of extremely powerful gravitational lenses was central to the success of the Frontier Fields project. We here briefly review the surveys that provided this crucial pool of targets, before focusing on the results of ongoing work to identify similarly extreme (if not more extreme) systems at higher redshift for the exploration of a yet more distant extragalactic frontier - with JWST and other, evermore ambitious missions.
\end{abstract}

Keywords. gravitational lensing, surveys, galaxies: clusters: general, X-rays: galaxies: clusters

\section{Introduction}

Progress in observational astronomy is attained through broad and/or deep studies of either blank fields or carefully selected individual targets. Clearly, the former kind of target is much easier to find than the latter. Consequently, projects like the Hubble Frontier Fields (HFF) rely heavily on the availability of not just a handful of possible targets but on a large pool of them, allowing the selection of objects that meet a wide range of criteria, from suitable intrinsic properties to advantageous location in the sky. For the HFF, these requirements could only be met thanks to prior, extensive searches for extremely powerful gravitational lenses, i.e., massive galaxy clusters. Since the projected surface density of these rare systems is a mere $0.005 \mathrm{deg}^{-2}$, the required sizable samples can be compiled only by means of (essentially) all-sky surveys.

\section{MACS}

The most important resource for the compilation of massive-cluster samples has been the ROSAT All-Sky Survey (RASS, Voges et al. 1999), or more specifically, the RASSbased Massive Cluster Survey (MACS; Ebeling et al. 2001, 2007, 2010; Mann \& Ebeling 2012) which contributed three of the six HFF targets and re-discovered two of the remaining three (Fig. 1). (Of the six HFF targets, only A370 is not a MACS cluster, owing to its low 3D mass and hence low X-ray luminosity - however, being a rare line-of-sight merger of two equally massive clusters, A370 is a powerful gravitational lens anyway.)

Detailed studies of galaxy clusters uniquely address a wide range of research topics (as demonstrated in this Focus Meeting), and the sample selected for the HFF, as well as the spectacular data collected by the HFF initiative, will satisfy many of the current scientific demands. The next frontier brings new challenges though. The strong focus on NIR and IR observations of JWST, as well as its location at L2, render many of the HFF targets less than ideal for observation by the next-generation space telescope. In particular high Ecliptic latitude has emerged as a new selection criterion, prompting a fresh search for additional, powerful cluster lenses. 


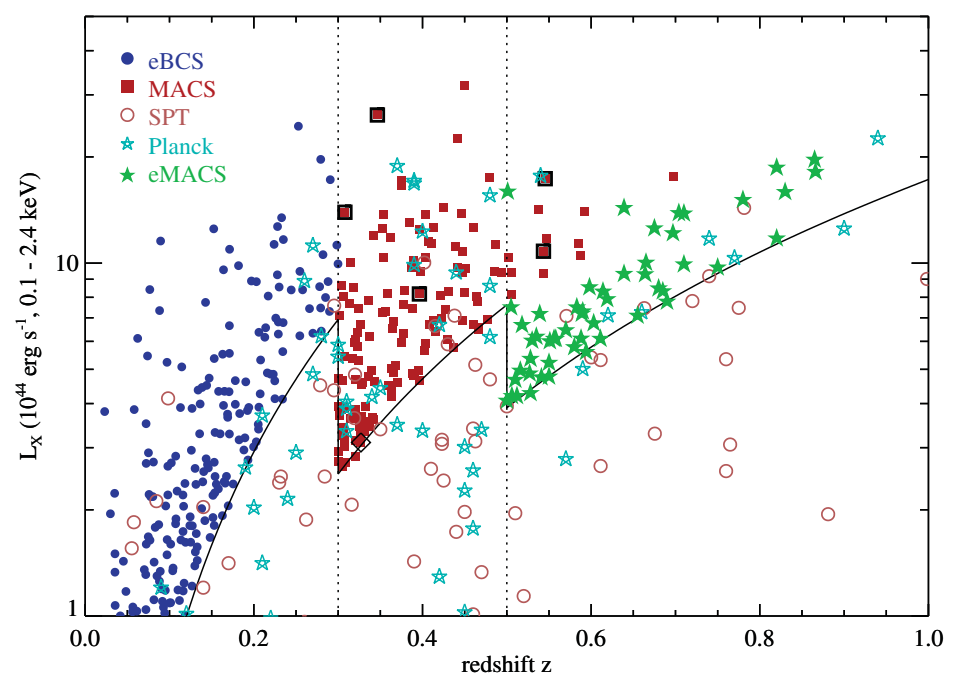

Figure 1. Distribution of various large-area cluster samples in redshift - X-ray luminosity space. The five MACS clusters selected as HFF targets are highlighted by black frames. We show all Planck clusters confirmed in the XMM verification phase as well as the SPT-SZ catalogs from Andersson et al. (2011) and Reichardt et al. (2013).

\section{3. eMACS}

Building on the success of MACS, the extended MACS project (eMACS; Ebeling et al. 2013) uses an even lower RASS X-ray flux limit and the recent availability of highquality 5-passband optical imaging from PanSTARRS-1 (Kaiser et al. 2007) to identify exclusively distant galaxy clusters $(z>0.5)$ among the over 65,000 RASS sources that fall into the almost $21,000 \mathrm{deg}^{2}$ of the eMACS survey area. The current eMACS sample is shown in Fig. 1, together with other cluster samples, both X-ray and SZ-selected. Note how the small solid angle of the SPT-SZ survey prevents it from detecting a larger number of truly massive clusters, while beam dilution reduces the Planck satellite's S/N on high-redshift clusters (Reichardt et al. 2013) to an extent that effectively requires $\mathrm{X}$-ray evidence to eliminate false-positive detections ("An SZ candidate with no signal at all in the RASS is false at very high confidence"; Planck Collaboration, 2013).

As targeted follow-up observations of eMACS clusters continue (including an HST SNAPshot programme), several highly promising strong-lensing clusters have been identified. Conducting a full, quantitative assessment of their suitability for JWST will be our next priority.

\section{Closing remarks}

Identifying the most promising clusters is an obviously indispensable prerequisite for the conduction of cluster-based research such as the one discussed in this meeting. Please give credit to those who facilitated your work by discovering the targets of your studies. eMACS is picking up where MACS left off, and we are confident that we will be able to provide the community with many more exceptional cluster targets in the very near future.

\section{References}

Andersson et al. 2011, ApJ, 738, 48

Ebeling et al. 2001, ApJ, 553, 668

Ebeling et al. 2007, ApJ, 661, L33

Ebeling et al. 2010, MNRAS, 407, 83

Mann \& Ebeling 2012, MNRAS, 420, 2120

Ebeling et al. 2013, MNRAS, 432, 62 
Kaiser et al. 2007, AAS, \#211, \#142.05

Reichardt et al. 2013, ApJ, 763, 127

Planck Collaboration 2013, A\&SA, 550, 130

Voges et al. 1999, A\&GA, 505, 991 